\title{
Antioxidant intervention in rheumatoid arthritis: results of an open pilot study
}

\author{
Richard M. van Vugt • Philip J. Rijken • \\ Anton G. Rietveld • Arno C. van Vugt • \\ Ben A. C. Dijkmans
}

Received: 2 January 2008 / Accepted: 11 January 2008 / Published online: 15 February 2008

(C) The Author(s) 2008

\begin{abstract}
There is evidence that reactive oxygen species play a causal role in auto-immune diseases, such as rheumatoid arthritis (RA). Despite the supporting evidence for a beneficial effect of antioxidants on clinical characteristics of RA, the right balance for optimal effectiveness of antioxidants is largely unknown. To determine the potential beneficial effects of an antioxidant intervention on clinical parameters for RA, an open pilot study was designed. Eight non-smoking female patients with rheumatoid factor + RA and a Disease Activity Score (DAS 28) higher than 2.5 were enrolled in the study. Patients had to be receiving stable non-steroidal anti-inflammatory drug treatment and/ or 'second line' medication for at least 3 months. The pilot group consumed $20 \mathrm{~g}$ of antioxidant-enriched spread daily during a period of 10 weeks. The intervention was stopped after 10 weeks and was followed by a 'wash-out' period of 4 weeks. At $t=0, t=10$ weeks and $t=14$ weeks, patients' condition was assessed by means of DAS. In addition, standard laboratory analyses were performed, and bloodsamples for antioxidants were taken. The antioxidantenriched spread was well tolerated. All laboratory measures of inflammatory activity and oxidative modification were generally unchanged. However, the number of swollen and
\end{abstract}

R. M. van Vugt $(\bowtie) \cdot$ B. A. C. Dijkmans

Department of Rheumatology and Clinical Immunology,

VU Medical University Centre,

P.O. Box 7057, 1007 MB Amsterdam, The Netherlands

e-mail: r.vanvugt@vumc.nl

\section{A. C. van Vugt}

Department of Rheumatology, Waldeck Pyrmontlaan 10, 1075 BW Amsterdam, Netherlands

\section{P. J. Rijken · A. G. Rietveld}

Unilever Health Institute, Unilever Research Vlaardingen,

Vlaardingen, The Netherlands painful joints were significantly decreased and general health significantly increased, as reflected by a significantly improved (1.6) DAS at $t=10$ weeks. The antioxidant effect was considered beneficial as, compared to the scores at $t=0$, the DAS significantly reduced at $t=10$ weeks. Increase of the DAS (0.7) after the "wash-out period" at $t=14$ confirmed a causal relation between changes in clinical condition and antioxidants. This open pilot study aimed to assess the clinical relevance of an antioxidant intervention as a first step in assessing potential beneficial effects of antioxidants on rheumatoid arthritis. These conclusions need to be validated in a larger controlled study population.

Keywords Antioxidants - Clinical parameters .

Intervention $\cdot$ Rheumatoid arthritis

\section{Introduction}

In spite of the great advances that have been made in the development of new drugs for the treatment of patients with rheumatoid arthritis (RA), many patients are interested in alternative treatments like dietary therapy. Although the aetiology of rheumatoid arthritis is still unknown, the inflammation resulting from the immunological reaction is quite well described. It is known that neutrophil granulocytes, macrophages and lymphocytes are activated, and that reactive oxygen and nitrogen species (RS) are produced [1, 2]. These RS can react with lipid, protein and nucleic acids and are thought to be of importance for the aetiology and chronicity of the inflammatory rheumatic diseases [3, 4]. One approach to counteract this oxidative stress situation is the use of antioxidants as therapeutic agents. There is some evidence for a positive effect of antioxidants on clinical symptoms of RA [5, 6]. However, this evidence is weak, 
and information about the most effective antioxidants, antioxidant doses or combinations is lacking The intervention studies that have been conducted have tested vitamin $\mathrm{E}$ exclusively $[6,7]$.

Against this background, an open (uncontrolled) pilot study was designed to assess if effects of antioxidants on the clinical parameters of patients with RA could be confirmed and if these effects would associate with changes in a selected number of molecular markers related to human antioxidant defence system status. Further, a mix of antioxidants was to be tested rather than a single high-dosed compound.

\section{Materials and methods}

The open study was approved by the ethical committee. Eight patients with rheumatoid factor $+\mathrm{RA}$, as defined by the ARA revised criteria were enrolled in the study. To avoid influences of gender, only female patients participated. Inflammatory disease activity was defined as a Disease Activity Score (DAS) higher than 2.5. Inclusion criteria included non-smoking and no serious co-morbidity. Patients had to be receiving stable non-steroidal antiinflammatory drug (NSAID) treatment and/or 'second line' medication for at least 3 months. Those taking antioxidant supplements were excluded from the study. During a period of 10 weeks, patients consumed $20 \mathrm{~g}$ of antioxidantenriched margarine daily. The spread contained a mix of a-tocopherol $(400 \mathrm{mg})$, lycopene $(10 \mathrm{mg})$, palm oil carotenoids (5 mg; mainly $\alpha$-carotene) and lutein $(10 \mathrm{mg})$. Further, patients received vitamin C (200 mg daily) as a supplement.

The intervention of 10 weeks was followed by a "washout' period of 4 weeks. At $t=0, t=5$ weeks, $t=10$ weeks and $t=14$ weeks, patients' condition was assessed by means of morning stiffness, visual analogue scale pain, general health score and DAS 28-score (including the following parameters: pain, swelling of joints, erythrocyte sedimentation rate (ESR), general feeling of well-being). At $t=0, t=10$ weeks and at $t=14$ weeks, blood was collected, and standard clinical laboratory analyses (ESR, C-reactive protein, hemoglobin, leucocytes, thrombocytes, serum values of creatinine and transaminases) were performed. In addition, blood antioxidant status and markers of antioxidant capacity [ferric reducing ability of plasma (FRAP)] and oxidative stress (8-epi F2-isoprostane) levels were determined. Determination of vitamin A, not present in the spread, was used as a control. Serum was collected immediately after blood sampling and stored in liquid nitrogen. The potential antioxidant effect was defined as beneficial if the DAS score would significantly improve $>1.2$ at $t=10$ weeks as compared to the scores at $t=0$. At $t=$

\section{DAS per patient}

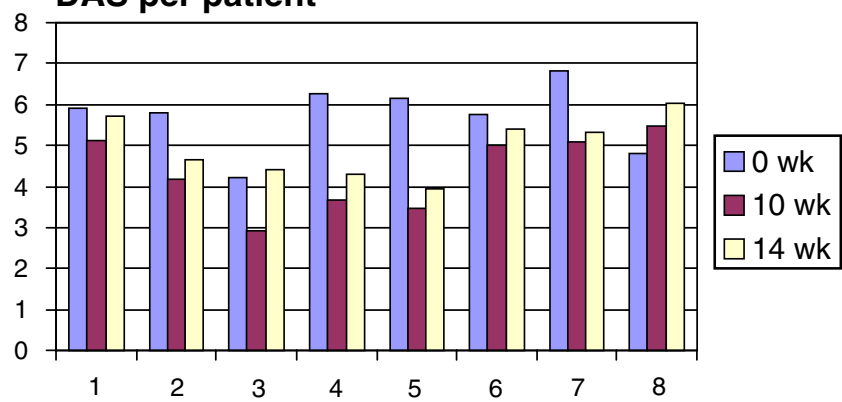

Fig. 1 Clinical condition by Disease Activity Score

14 weeks, a 'wash-out" effect was assessed using the same parameters. Return of the clinical condition of the pilot group to that at $t=0$ may indicate a causal relation between changes in clinical condition and antioxidant intake, although a placebo-controlled intervention would be better required for this assessment.

Statistical analyses were done using a paired $t$ test.

\section{Results}

The antioxidant-enriched spread was well tolerated. None of the patients withdrew from the study because of adverse reactions. The number of swollen and painful joints were significantly decreased, and general health significantly increased at $t=10$ weeks. This was shown by a significantly decreased DAS $(-1.6)$ at 10 weeks. After the "wash-out period", the DAS score was increased again by 0.6 (Figs. 1 and 2).

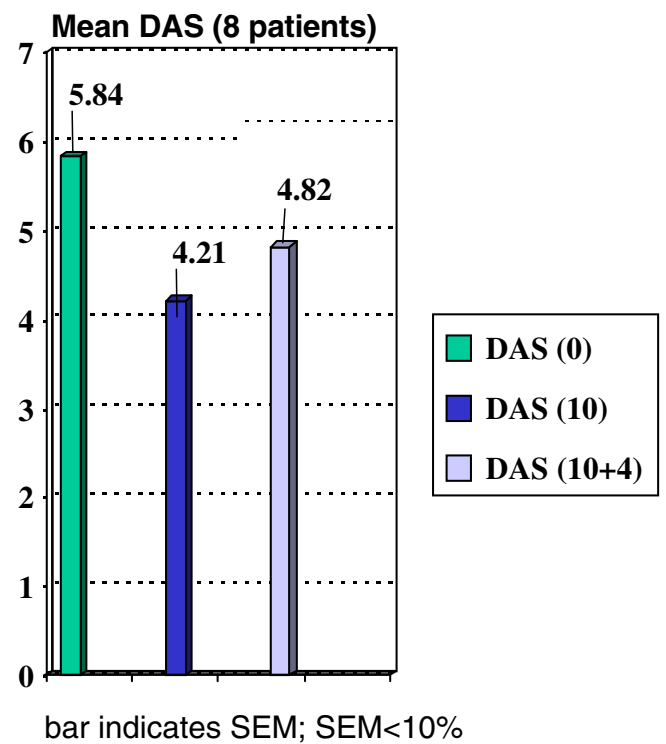

Fig. 2 Mean Disease Activity Score 
plasma vitamin $E(\mathrm{ug} / \mathrm{ml})$

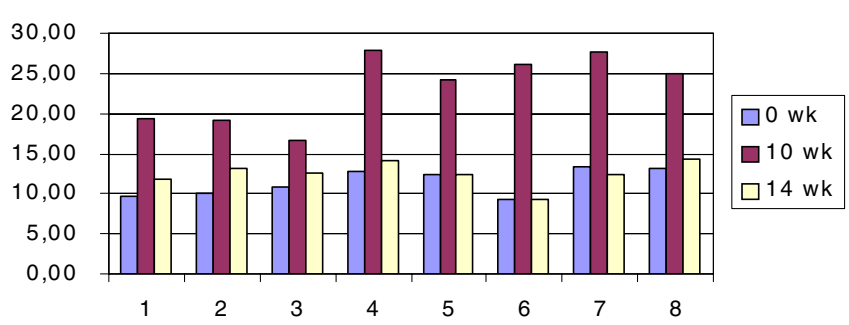

plasma lycopene $(\mathrm{ng} / \mathrm{ml})$

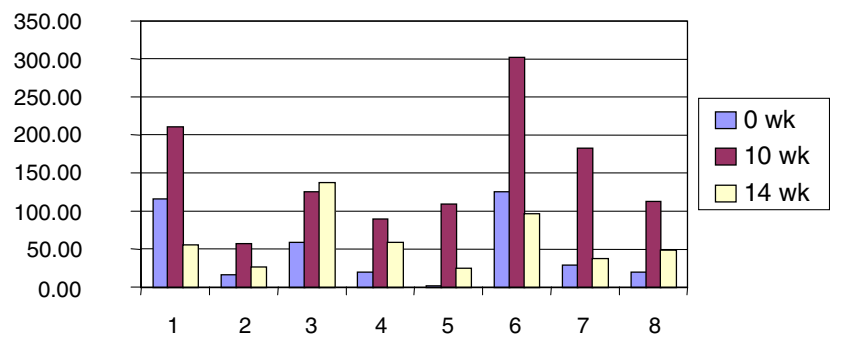

plasma vitamin C

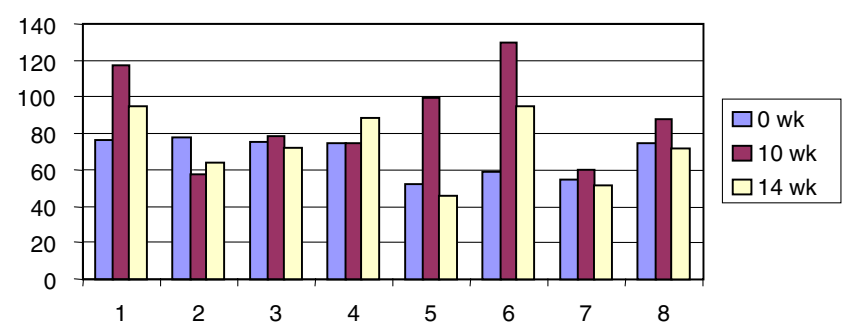

Fig. 3 Plasma antioxidant status in eight patients plasma lutein $(\mathrm{ng} / \mathrm{ml})$

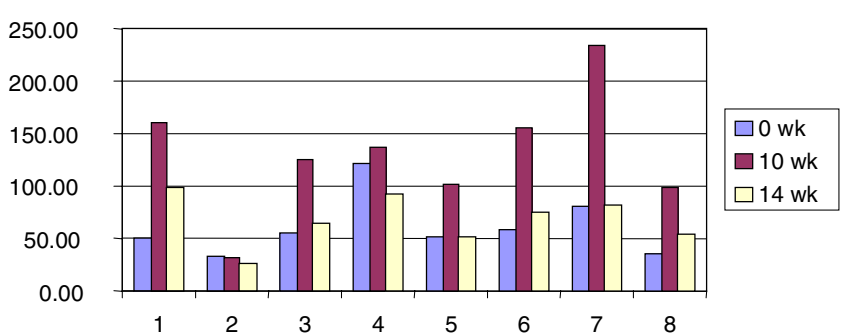

plasma $\alpha$-carotene $(\mathrm{ng} / \mathrm{ml})$

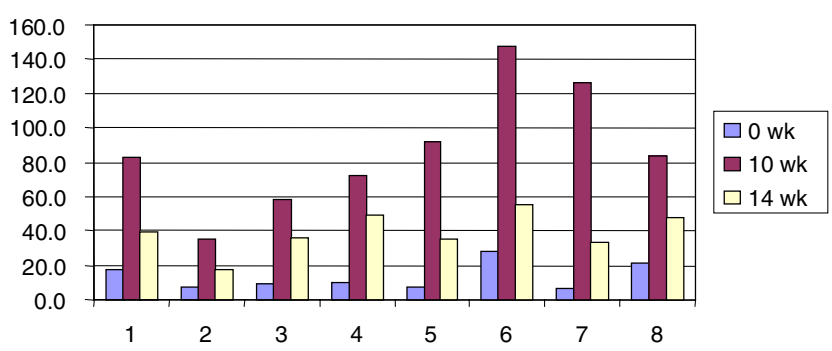

Fig. 4 Markers of antioxidant capacity and oxidative stress
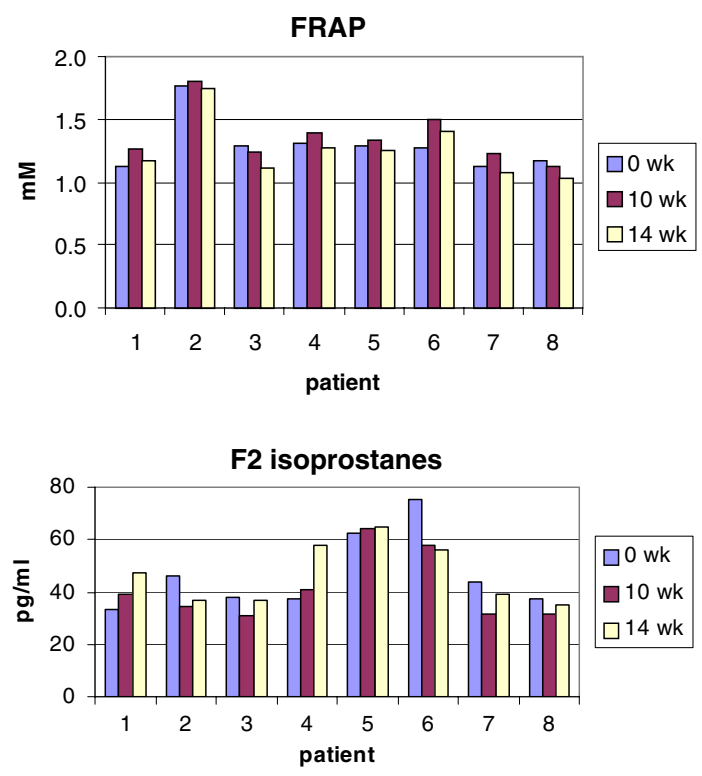

average relative FRAP

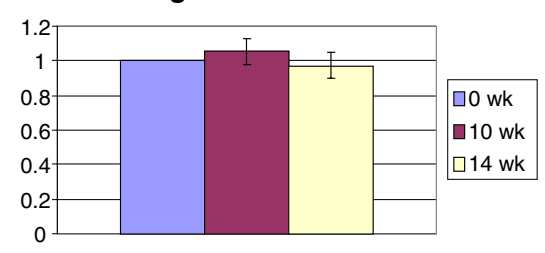

average relative F2-isoprostanes

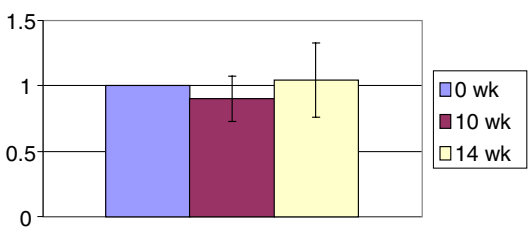


The standard laboratory measures of inflammatory activity were relatively unchanged. Levels of plasma vitamin E, lutein, lycopene and alpha-carotene were significantly increased at 10 weeks. No significant changes were seen in vitamin A and vitamin C levels (Fig. 3).

Markers of antioxidant capacity and oxidative stress displayed trends for differences, but none of these reached statistical significance (Fig. 4).

\section{Discussion}

This pilot study was conducted to obtain some first indications for potential beneficial effects of an antioxidant intervention on clinically relevant parameters for rheumatoid arthritis. In our present study, a significant reduction in Disease Activity Score (DAS) was observed following antioxidant intervention of 10 weeks. This was accompanied by significant increases in blood levels of antioxidants administered. The reduction of DAS is remarkable as all patients had an active (high initial mean DAS of 5.8) longstanding disease and had tried all kinds of diseasemodifying anti-rheumatic drugs, including combination therapy. The unchanged level of vitamin A, not present in the spread, reinforces the significance of the increased levels of the blood antioxidant status. As NSAIDs can influence the absorption of vitamin $\mathrm{C}$, this could explain the non-significant changes of the serum concentration of this antioxidant.

A limited number of clinical intervention studies is available which support the therapeutic or prophylactic activity of antioxidants in the pathogenesis of RA. These studies were all conducted with an oral intake of $1,200 \mathrm{mg} /$ day of vitamin E. Edmonds et al. [6] reported that the antioxidant a-tocopherol significantly reduced pain parameters in a placebo-controlled double-blind trial following a 3 -week supplementation period. Results from a randomised double-blind parallel group comparison study with atocopherol and diclophenac showed that the clinical parameters assessed, e.g. morning stiffness, Ritchie joint index, grip strength and pain, were significantly reduced by vitamin E, with similar effectivity and less side effects as compared to regular drug therapy by diclofenac [7]. Results from other intervention studies have generally been in line with these observations $[8,9]$.

In our study, not only the effect of vitamin $\mathrm{E}$ on the inflammatory response and clinical symptoms was evaluated but also the interactions with bioavailable natural antioxidants such as carotenoids, vitamin $\mathrm{C}$ and others. In view of the limited number of controlled studies, the supporting evidence for beneficial effects of antioxidants on clinical characteristics of rheumatoid arthritis may be considered limited but promising. Another point is that the potential mechanism of action of antioxidants in rheumatoid arthritis needs further attention. The aspect of damage by RS and loss of critical functions can be analysed by use of 'molecular markers'. These markers may include antioxidant status, products which arise as a consequence of oxidative damage to lipid, protein and DNA and tissue damage (as assessed by lactic dehydrogenase release) or cytokine levels (cq anti-TNF $\alpha$ ).

Although markers are very informative from a mechanistic point of view, a drawback is that none of them has been validated against clinical conditions of patients with RA. In this study, a trend for reduced levels of measures of antioxidant capacity and of 'footprints' of oxidative stress was observed, e.g. statistically non-significant increase in FRAP and reduction in F2-isoprostane levels. Furthermore, these markers showed a trend for correlation with clinical conditions. The data indicate that statistically significant effects may be observed when the number of patients enrolled is increased.

This open pilot study therefore should be viewed as a first step in assessing potential beneficial effects of the antioxidants tested on rheumatoid arthritis. The data indicate there may be potential in using mixes of antioxidants in the treatment of RA alongside drug treatment so that drug dosage may be reduced while hopefully retaining efficacy. Further, multi-drug/antioxidant therapy could be used to reduce drug dose and thus side effects of treatment.

In conclusion, our present pilot study indicated that intervention with antioxidant-enriched margarine in RA results in consistent and significant relief of clinical symptoms. Furthermore, increases in blood antioxidant status and indications for effects on oxidative stress markers were observed. These data are promising and indicate need for a double-blind, placebo-controlled randomised human trial to establish effect and demonstrate causality.

Open Access This article is distributed under the terms of the Creative Commons Attribution Noncommercial License which permits any noncommercial use, distribution, and reproduction in any medium, provided the original author(s) and source are credited.

\section{References}

1. Lunec J, Halloran SP, White AG, Dormandy L (1981) Freeradical oxidation (peroxidation)products in serum and synovial fluid in rheumatoid arthritis. J Rheumatol 8:233-245

2. McInnes IB, Leung BP, Field M, Wei XQ, Huang FP, Sturrock RD, Kinninmonth A, Weidner J, Mumford R, Liew FY (1996) Production of nitric oxide in the synovial membrane of rheumatoid and osteoarthritis patients. J Exp Med 184:1519-1524

3. Remans PH, van Oosterhout M, Smeets TJ, Sanders M, Frederiks WM, Reedquist KA, Tak PP, Breedveld FC, van Laar JM (2005) Intracellular free radical production in synovial $\mathrm{T}$ lymphocytes 
from patients with rheumatoid arthritis. Arthritis Rheum 52 (7):2003-2009 July

4. Sklodowska M, Gromadzinska J, Biernacka M et al (1996) Vitamin $\mathrm{E}$, thiobarbituric acid reactive substance concentrations and superoxide dismutase activity in the blood of children with juvenile rheumatoid arthritis. Clin Exp rheumatol 14:433-439

5. Oliver JE, Silman AJ (2006) Risk factors for the development of rheumatoid arthritis. Scand J Rheumatol 35(3):169-174 May-Jun

6. Edmonds SE, Winyard PG, Guo R, Kidd B, Merry P, LangrishSmith A, Hansen C (1997) Putative analgesic activity of repeated oral doses of vitamin $\mathrm{E}$ in the treatment of rheumatoid arthritis.
Results of a prospective placebo-controlled double blind trial. Ann Rheum Dis 56:649-655

7. Wittenborg A (1998) Effectiveness of vitamin E in comparison with diclofenac sodium in treatment of patients with chronic polyarthritis Z. Rheumatol 57(4):215-221

8. Jaswal S, Metha HC, Sood AK, Kaur J (2003) Antioxidant status in rheumatoid arthritis and role of antioxidant therapy. Clin Chim Acta 338(1-2):123-129

9. Hagfors L, Leanderson P, Skoldstam L, Anderson J, Johanson G (2003) Antioxidant intake, plasma antioxidants and oxidative stress in a randomized, controlled, parallel, Mediterranean dietary intervention study on patients with rheumatoid arthritis. Nutr J 2:5 july 30 